\title{
Fungi form interkingdom microbial communities in the primordial human gut that develop with gestational age
}

\author{
Kent A. Willis ${ }^{1}$, John H. Purvis ${ }^{1}$, Erin D. Myers ${ }^{2}$, Michael M. Aziz ${ }^{4}$, Ibrahim Karabayir ${ }^{5}$, Charles K. \\ Gomes $^{3}$, Brian M. Peters ${ }^{6}$, Oguz Akbilgic ${ }^{5}$, Ajay J. Talati ${ }^{1,4}$, Joseph F. Pierre ${ }^{3,8 *}$ \\ ${ }^{1}$ Department of Pediatrics, Division of Neonatology, College of Medicine, The University of Tennessee \\ Health Science Center (UTHSC), Memphis 38103 TN, USA \\ ${ }^{2}$ College of Medicine, UTHSC \\ ${ }^{3}$ Deparment of Pediatrics, Obesity, College of Medicine, UTHSC \\ ${ }^{4}$ Department of Obstetrics and Gynecology, College of Medicine, UTHSC. \\ ${ }^{5}$ Department of Health Informatics and Data Science, Stritch School of Medicine, Loyola University \\ Chicago, Chicago 60660 IL, USA \\ ${ }^{6}$ Department of Clinical Pharmacy and Translational Science, College of Pharmacy, UTHSC \\ ${ }^{7}$ Center for Health Outcome and Informatics Research, Loyola University Chicago, Chicago, IL, USA \\ ${ }^{8}$ Deparment of Microbiology, Immunology and Biochemistry, College of Medicine, UTHSC \\ ${ }^{*}$ Correspondence and request for materials should be addressed to J.F.P. (email: \\ jpierre1@uthsc.edu)
}

Keywords Meconium; Microbiome; Mycobiome; Premature birth; Gut ecology; Dectin-1

\section{Abstract}

Fungal and bacterial commensal organisms play a complex role in the health of the human host.

Expansion of commensal ecology after birth is a critical period in human immune development.

However, the initial fungal colonization of the primordial gut remains undescribed. To investigate

primordial fungal ecology, we performed amplicon sequencing and culture-based techniques of firstpass meconium, which forms in the fetal intestine prior to birth, from a prospective observational cohort of term-born and preterm newborns. Here, we describe fungal ecologies in the primordial gut that develop complexity with advancing gestational age at birth. Our findings suggest homeostasis of fungal commensals may represent an important aspect of human biology present even before birth. Unlike bacterial communities which gradually develop complexity, the domination of the fungal communities of some preterm infants by Saccromycetes, specifically Candida, may suggest a pathologic association with preterm birth. 


\section{Introduction}

Human health is intimately entwined with the commensal microorganisms that constitutionally colonize our barrier surfaces, such as the intestinal tract. The human gut microbiome is host to a complex interkingdom ecology of bacteria, fungi, archaea, protozoa and viruses. While recent research has led to a drastic expansion in our understanding of bacterial communities in the gut ${ }^{1-4}$, little is known about the role of fungal organisms, collectively the mycobiome, particularly in relation to their impact on the population dynamics of early-life colonization.

The tightly choreographed maturation of commensal microbial communities is crucial to mammalian immune development ${ }^{5}$. Fungal organisms can form a crucial nidus for initial colonization ${ }^{6}$ and may play a vital role in facilitating early colonization of the neonatal gut. Disruption of this process could produce pathogenic fungal overgrowth or invasive disease. Indeed, this mechanism may play a role in the development of asthma and other metabolic and immunological diseases ${ }^{7}$. The intestinal mycobiota likely confers unique physiologic effects to the host, in part by processing nutrients and vitamins either separately or in conjunction with the microbiome. However, characterization of the functional impact of early mycobiome is impaired by limited knowledge of the normal development of these communities. To understand the functional impact of the early-life mycobiome on human health, it is necessary to determine if the human primordial gut hosts fungal communities, if these communities are established before or after birth and if the presence of fungi in the early gut impacts neonatal physiology.

Advances in next-generation sequencing techniques have pushed back the timing of initial commensal colonization ${ }^{3}$ which was initially thought to occur at birth ${ }^{8,9}$. Despite these advances, interkingdom interactions within microbial communities and the influence of environmental factors on the dynamics of microbial ecosystems remains poorly understood. Due to delayed progress in sequencing and characterizing fungal communities ${ }^{10}$, the appreciation of commensal fungal ecology 
67 and biomedical impact of fungi lags behind the bacterial microbiome. It appears that during the first

68 several years of life, early fungal communities, like their bacterial counterparts, arise from maternal

69 communities, but unlike their bacterial counterparts, remain at a relatively low and consistent

70 diversity ${ }^{11,12}$. The relative consistency of early mycobiome makes understanding initial colonization

71 important to unravel both the long-term biomedical impact of fungal communities and to explain their

72 divergence from bacterial communities. While we are beginning to understand the development of the

73 mycobiome during early-life, the timing of initial fungal colonization remains undescribed.

75 To characterize the interkingdom ecologies of the fetal microbiome and understand when fungal

76 colonization first occurs, we performed a molecular and culture-based survey of first-pass meconium

77 collected from an observational cohort of preterm and term-born human newborns. In this study, we

78 characterize fungal communities in first-pass meconium within hours of birth and explore the influence

79 of perinatal factors on the foundational ecology of the human gut, laying the groundwork for

80 understanding the functional impact of fungal communities on the host. We support our observations

81 by examining the fetal and neonatal mouse mycobiome. We describe complex bacterial and fungal

82 commensal communities that develop in abundance and diversity with length of gestation. Our results

83 suggest that the human fetus is naturally exposed an increasing variety of microbial DNA as gestation

84 progresses. However, the increased prevalence of fungal order Saccharomyces, especially the genus

85 Candida, in preterm infants may also suggest a pathologic association with preterm birth.

\section{Results}

\section{Study design and cohort characteristics}

To characterize the fungal ecology of the primordial human gut, we collected the first-pass meconium

93 from a cohort of very low birthweight preterm (<1500 g, gestational age 23-32 weeks, $\mathrm{n}=54)$ and

94 term-born (gestational age $37-41$ weeks, $n=36$ ) newborns and characterized the microbial

95 communities using both culture-independent and culture-dependent techniques (Fig. 1). Of these 90 
infants prospectively enrolled from a Level IIIB Neonatal Intensive Care Unit and Well Baby Nursery at

97 single academic medical center, 19 infants (31.5\% of preterm and $5.6 \%$ of term-born infants, $\chi^{2} p$

$98=0.0033$ ) did not produce a meconium stool that could be collected during the first $48 \mathrm{~h}$ of life (Fig.

99 1a). To differentiate the effects of perinatal factors on microbial ecology, we prospectively collected

100 clinical data describing perinatal exposures (Fig. 1b). In addition to lower gestational age, infants born

101 preterm were more likely than term-born infants to have a lower birthweight, a lower Apgar and higher

102 Critical Risk Index for Babies II (CRIB-II) scores, receive postnatal antibiotics and be delivered by

103 cesarean section (Table 1).

104

105

113

114

115

116

117

118

119

\section{Figure 1}

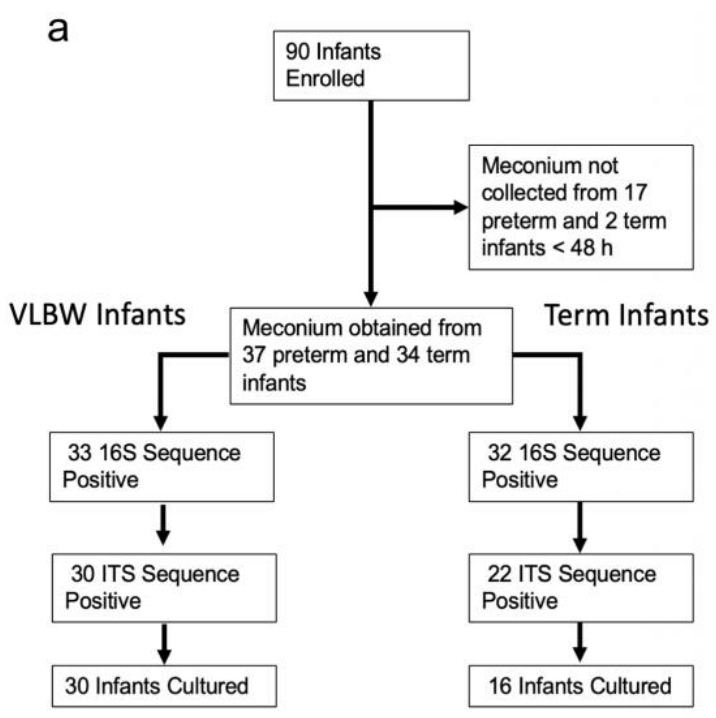

Fig. 1. Curation of clinical and microbial data. a Schematic of patient allocation. VLBW, very low birthweight (preterm infants $<1500 \mathrm{~g}$ ). b Schematic of data acquisition and analysis. Low biomass samples of meconium, an intralumenal material formed in the intestine prior to birth, were analyzed by culture-based techniques or Illumina MiSeq and quantitative PCR after high-fidelity DNA extraction. Sequence reads were subjected to exacting data processing to remove potential contaminants. Clinical demographics were used to perform ecological modeling of microbial communities and develop sequential rarified random forest classifier machine learning models. A mouse model of normal pregnancy was utilized to quantify intrauterine fungal biomass. 16S rRNA, 16S ribosomal RNA (bacteria and archaea). ITS rDNA, internal transcribed spacer ribosomal DNA (fungi). 
121 Table 1. Cohort Characteristics

\begin{tabular}{llll}
\hline & Preterm & Term & $P$ Value \\
\hline Stooled $<48 \mathrm{~h}$ & 37 & 34 & \\
Male Sex & $49 \%$ & $56 \%$ & 0.5580 \\
Black/African American & $78 \%$ & $91 \%$ & 0.1361 \\
Maternal Age (yr) & $26.94 \pm 6.00$ & $25.15 \pm 4.64$ & 0.1731 \\
Gestational Age (wk) & $27.51 \pm 2.71$ & $39 \pm 1.15$ & $<0.0001$ \\
Birthweight (g) & $874.1 \pm 266.81$ & $3115 \pm 360.06$ & $<0.0001$ \\
CRIB-II Score & $10(8: 13)$ & $0(0: 0)$ & $<0.0001$ \\
Apgar Score (5 min) & $6(5: 9)$ & $9(9: 9)$ & $<0.0001$ \\
Cesarean Delivery & $68 \%$ & $35 \%$ & 0.0058 \\
Prenatal Antibiotics & $32 \%$ & $38 \%$ & 0.5987 \\
Prenatal Antibiotic Doses* $(\mathrm{n})$ & $1(1: 1.5)$ & $2(1: 2)$ & 0.2231 \\
Prenatal Antibiotic Doses $(\mathrm{n})$ & $0(0: 1)$ & $0(0: 1)$ & 0.7943 \\
Postnatal Antibiotics & $92 \%$ & $0 \%$ & $<0.0001$ \\
16S rRNA Positive & $92 \%$ & $94 \%$ & 0.5397 \\
ITS rDNA Positive & $88 \%$ & $65 \%$ & 0.0224 \\
\hline
\end{tabular}

122

123

124

125

126

127

128

130

131

132

${ }^{*}$ Number of doses received prenatally in those who received antibiotics. $n$ (quartile $25 \%$ : quartile $75 \%$ ) or $n \pm S D$.

\section{Fungal community composition differs by gestational age}

The bacterial community composition of the microbiome differs by gestational age at birth ${ }^{13}$. This may be related to differences in initial meconium colonization ${ }^{3}$. To explore if fungal colonization of the microbiome was influenced by gestational age, we performed a binary comparison of infants born at $<33$ weeks' $(n=37)$ and infants $>33$ weeks' gestation $(n=34)$. Meconium samples from preterm infants were more likely to contain fungal DNA than samples from term-born infants using internal transcribed spacer region 2 ribosomal DNA (ITS2 rDNA) amplicon sequencing at a read depth of 300x (88 versus $65 \%, \chi^{2} p=0.0224$, Table 1 ). The alpha diversity of these fungal sequences clearly increased with increasing gestational age (Shannon diversity index, ANOVA $f=4.5 p=0.04$. Principal coordinates analysis (PCoA) of Bray-Curtis dissimilarity matrices demonstrated fungal community structure was different by permutational multivariate analysis of variance $\left(\right.$ PERMANOVA, $R^{2}=0.308 p$ $=0.01$, Fig. 2c). Discriminant analysis of principal components suggests that the principal variables at the genus level are the prevalence of Elmerina, Cladosporium, Candida and Stereum. Similarly, canonical correspondence analysis $\left(\mathrm{CCA}, \chi^{2}\right.$ distance $\left.=0.71 f=1.14 p=0.004\right)$, and redundancy analysis $(\mathrm{RDA}$, variance $=3.83 f=1.22 p=0.001$ ) were both consistent with a clear differentiation in fungal community structure by advancing gestational age (Fig. 3d). 
143 Network analysis supports the sparseness of fungal communities, particularly in preterm infants,

144 which is likely related to the low fungal diversity (Fig 2d). However, as quantified by positive

145 Spearman's correlations, fungal networks do appear to develop complexity with increasing gestational 146 age.

148 The relative composition of the fungal communities varied with gestational age (Fig. 2 and 3). At the

149 order level, preterm infants were primarily colonized with Saccharomycetales (73.4\%), with other

150 fungal taxa accounting for less than a quarter of assigned reads. Term-born infants possessed

151 mycobiomes that were less dominated by a singles species than preterm infants. Saccharomycetales

152 and Polyporales accounted still accounted for about half of the fungal burden in infants with a later

153 gestation (22.1\% and $27.7 \%$, respectfully), but other minor taxa were more markedly prevalent with

154 increasing gestational age. In addition to Polyporales $(5.2 \%<33$ weeks' and $22.1 \%>33$ weeks'

155 gestation), Russulales showed the most pronounced expansion with increasing gestational age $(0.8 \%$

$156<33$ weeks' and $14.5 \%>33$ weeks' gestation, Fig. 2f, 3a). The most significant expansions in

157 abundance at the genus level were in Stereum, Malassezia, Elmerina and Armillaria. In contrast to

158 this trend, the domination of the mycobiome by Candida in some preterm infants lead to this particular

159 genus being uniquely more abundant in preterm infants overall. Finally, core microbiome analysis also

160 supports the expansion of fungal communities with increasing gestational age, with the addition of 2

161 unique core phyla and 3 unique core OTUs in term-born as compared to preterm infants (Fig. 3e).

162 To further investigate the association of specific fungal taxa with gestational age, we performed linear

163 discriminant analysis of effect size (LEfSe) to identify fungal taxa that could perform as high-

164 dimensional biomarkers for prematurity. The prevalence of the fungal order Saccharomyces and

165 specifically the genus Candida reliably identified preterm samples. In addition, the prevalence of

166 fungal class Elmerina and the genera Stereum and Malassezia reliably identified samples from full-

167 term newborns (Supplemental Fig. 3i). 
a
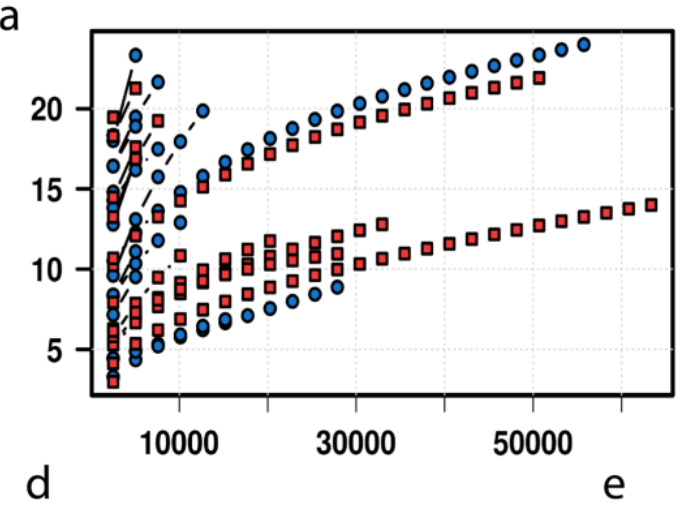

Figure 2

b
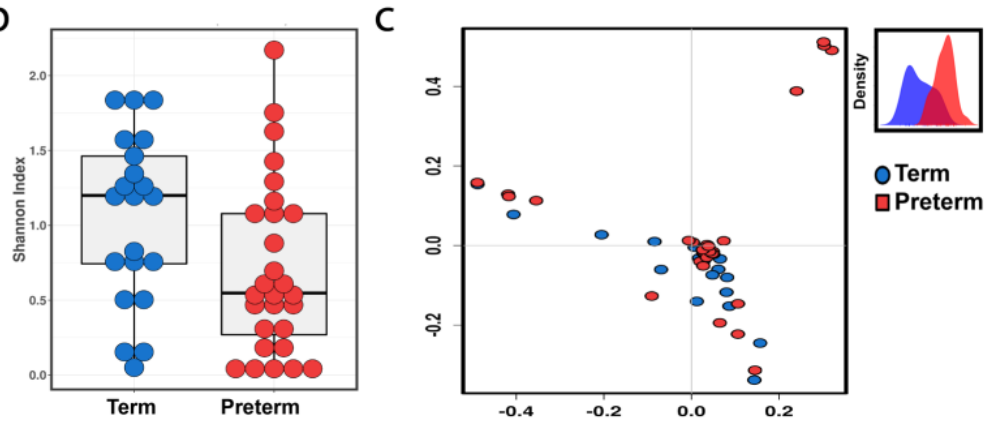

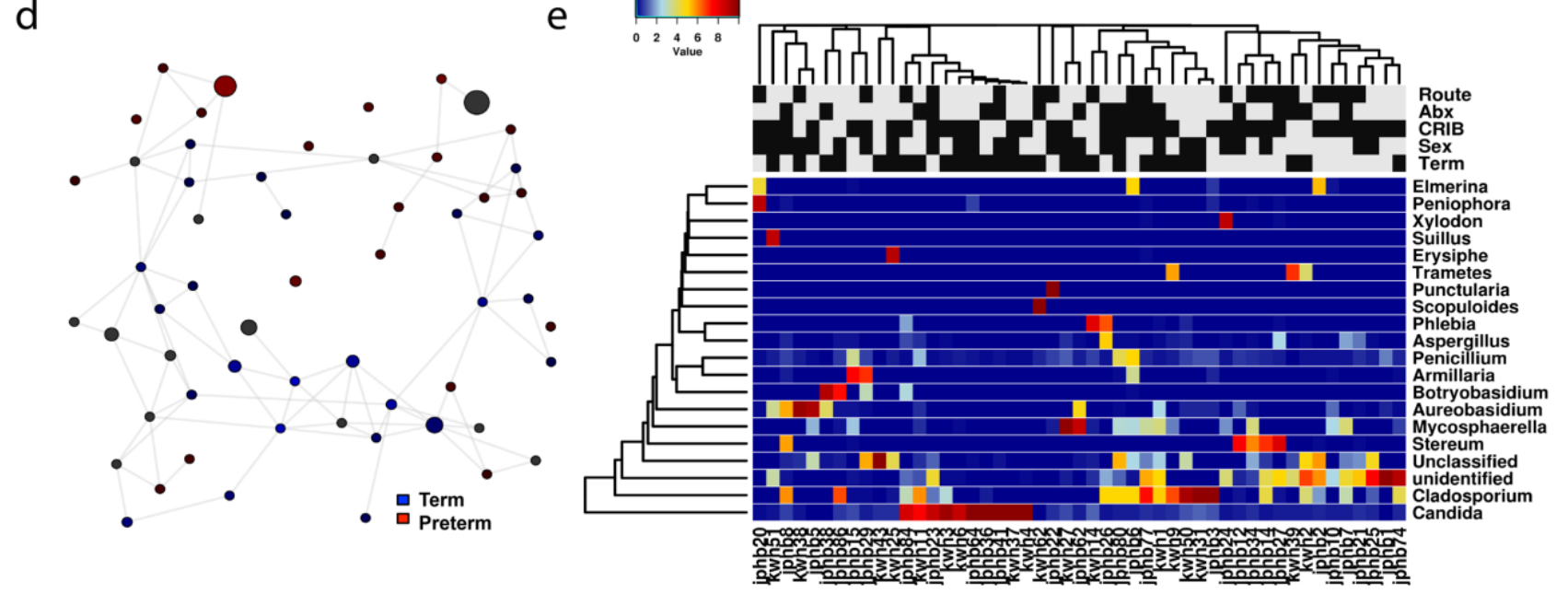

f

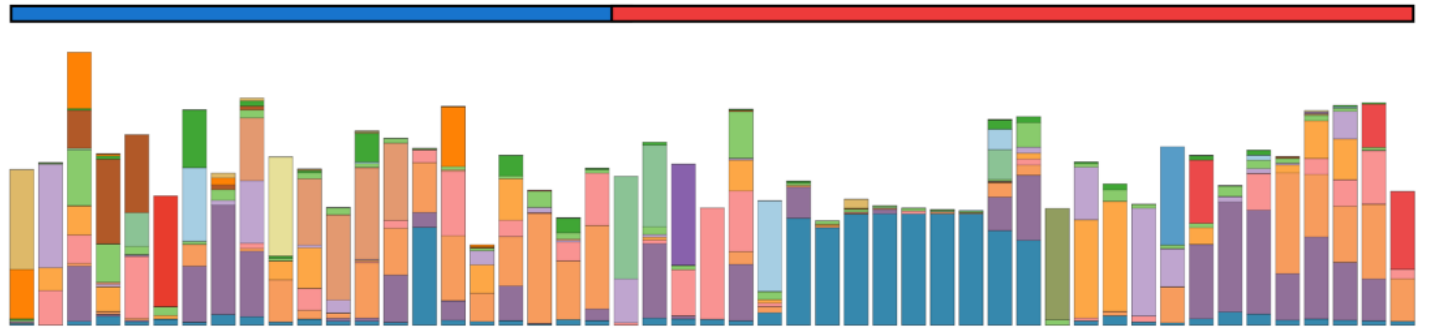

Fig. 2. Fungal ecologies in human meconium vary by the gestational age at birth.

b Alpha diversity quantified by the Shannon index, ANOVA $f=4.5 p=0.04$. $=0.01$. The subset displays a discriminant analysis of principal components. d Network analysis developed using Pearson's correlations. Positive correlations with false discovery rate-adjusted, $p$-values $<0.05$ are presented as an edge. e Heat map of differential distribution of taxa at the order level, ranked by gestation. f Relative abundance of taxa at the phylum level. Sqrt(TSS), square root total sum normalization 179 (Hellinger transformation).

180 For all analyses $n=71$. Preterm samples are displayed in red and term-born samples in blue. 
183

184

a

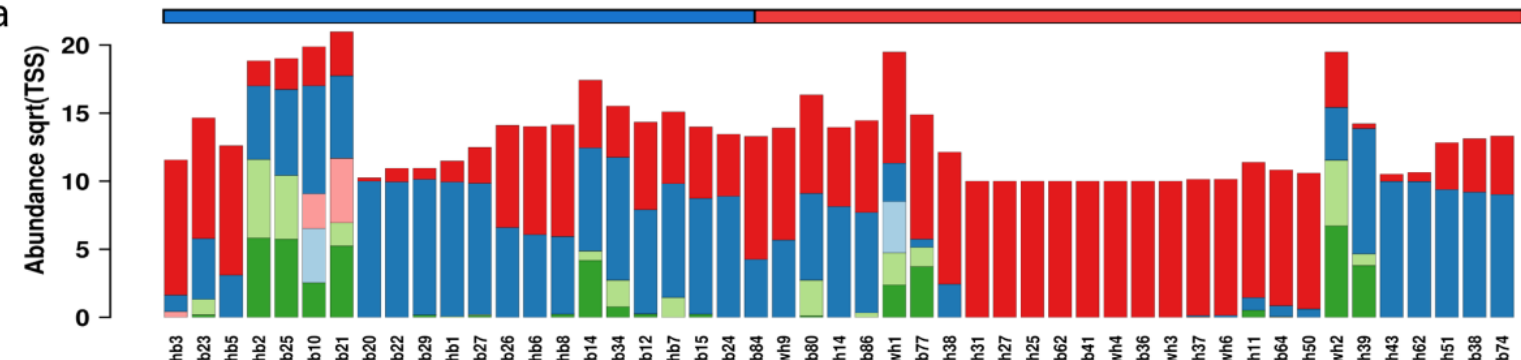

b

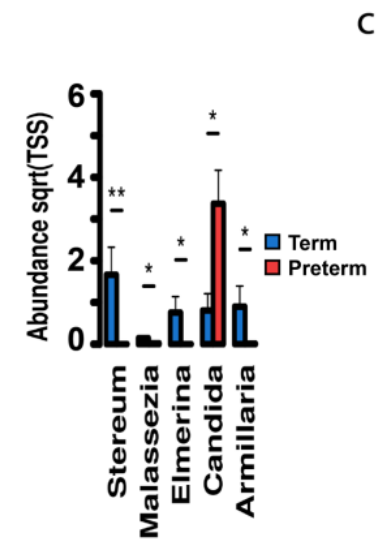

d

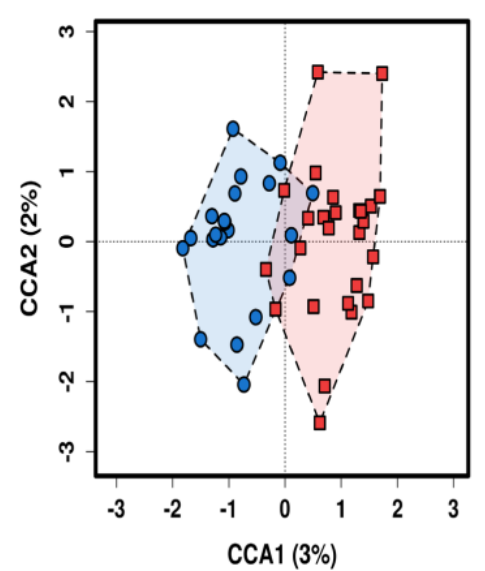

Figure 3

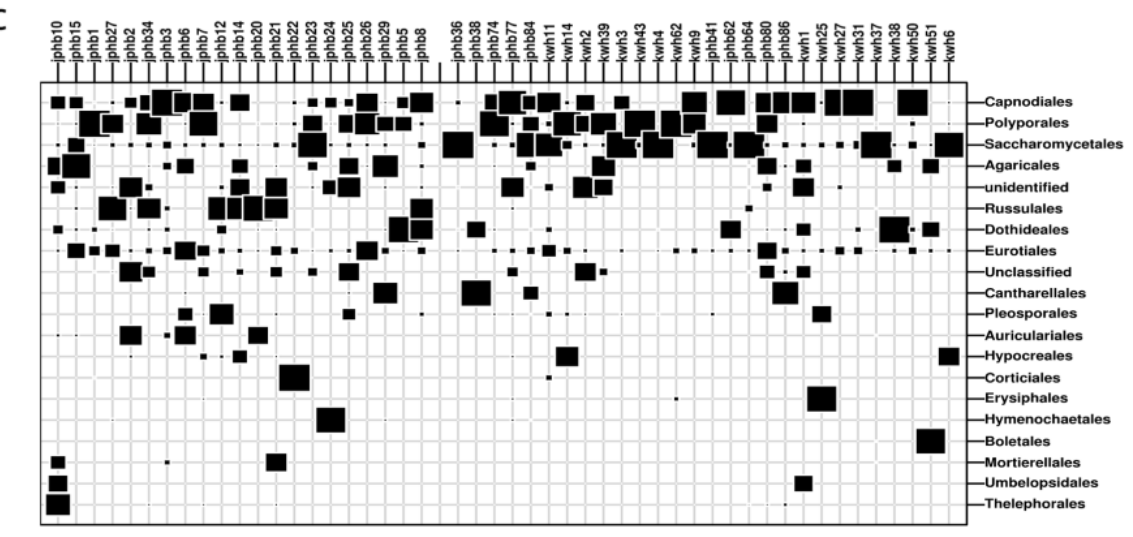

e

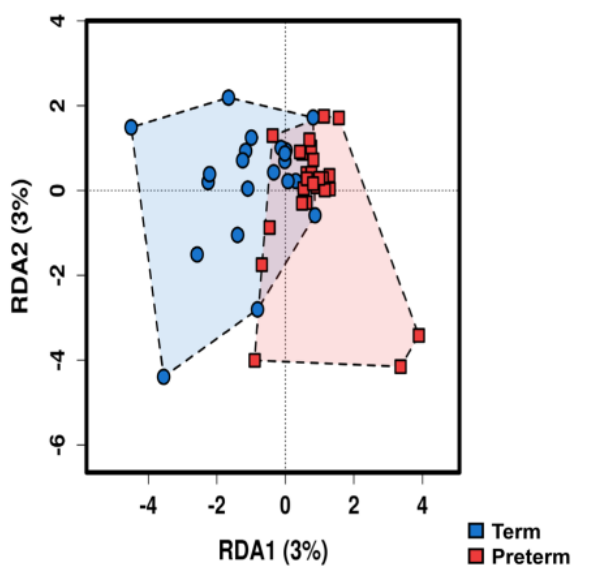

$\square$ Term

$\square$ Preterm

Features:
Ascomycota
Basidiomyco

Mortierellomycota Mucoromycota 品 Unclassified
185

Fig. 3. Fungal communities increase in complexity with advancing gestational age at birth. a Relative abundance at the phylum level. Sqrt(TSS), square root total sum normalization (Hellinger transformation).

b Distribution of key taxa at the genus level. ANOVA, Bonferroni, ${ }^{*} p<0.05,{ }^{* *} p<0.01$.

c Twenty most abundant interkingdom taxa at the order level.

d Canonical correspondence analysis (CCA), $\chi^{2}=0.71 f=1.14 p=0.004$, and redundancy analysis (RDA), variance $=3.83 f=1.22 p=0.001$.

e Core interkingdom OTUs and unique phyla between preterm and term-born infants. OTU, operational taxonomic unit.

Data are median \pm IQR $(n=71)$. Preterm samples are displayed in red and term-born samples in blue. 
198 In childhood, fungal colonization has been shown to be influenced by host sex ${ }^{14}$. We examined fungal

199 community composition in our cohort to see if fungal populations in meconium were influence by the

200 sex of the host. Alpha diversity of was unaltered by sex (Supplemental Fig. 4g). Relative abundance

201 differences were most pronounced for Polyporales (24.1\% in females and only $6.9 \%$ in males), with

202 other orders having less dramatic differences (Supplemental Fig. 4h). Meconium fungal community

203 structure was not influenced by host sex (PCoA, PERMANOVA $R^{2}=0.0209 p=0.529$, Fig. $4 a$ ).

204 Similarly, redundancy analysis (RDA, variance $=3.19 f=1.02 p=0.348$ ) was also not significant.

205

206

Antibiotics can have a profound effect on the compositional structure of the microbiome ${ }^{15}$, and lead to

changes in the fungal colonization as well ${ }^{16}$. To characterize the potential effect of maternal antibiotic exposure on the initial structure of the mycobiome, we examined how any antibiotic exposure altered fungal composition of the meconium. In our samples, perinatal antibiotic exposure did not produce significant differences in alpha diversity (Supplemental Fig. 4d). The relative abundance of

211 Saccharomycetales was increased by maternal antibiotic exposure $(60.1 \%$ after antibiotic exposure,

212 and $32.5 \%$ without antibiotic exposure, Supplemental Fig. 4e). The distribution of other major taxa

213 was similar. Fungal community structure was not significantly altered by prenatal antibiotic exposure

214 (PERMANOVA $R^{2}=0.202 p=0.598$ and RDA, variance $=3.51 f=1.12 p=0.083$, Fig. $4 b$ ).

216 Whether mode of delivery influences microbial colonization remains controversial ${ }^{2,17}$. To explore

217 influence of the delivery mode on fungal colonization we examined fungal community structure using

218 cesarean or vaginal delivery as a binary variable. Alpha diversity was not significantly altered by mode

219 of delivery, although cesarean born infants did demonstrate more variability (Supplemental Fig. 4a).

220 Relative composition differences showed some variation by mode of delivery (Supplemental Fig. 4b)

221 Similar to preterm infants, samples from infants born via caesarean were predominantly colonized by

222 Saccharomycetales (61.7\% after cesarean and 39.7\% after vaginal). Both groups expressed similar

223 levels of Polyporales (15.3\% after cesarean and $13.8 \%$ after), but Russulales was more prevalent in 
224 vaginally-born infants (13.2\% after vaginal and $0.62 \%$ after cesarean). While preterm infants were

225 more likely to be delivered by cesarean in our cohort ( $68 \%$ versus $35 \% \chi^{2} p=0.0058$, Table 1 ), as

226 characterized by PCoA, fungal community structure did not significantly differ after either vaginal or

227 cesarean delivery (PERMANOVA $R^{2}=0.202 p=0.598$ and RDA, variance $=3.51 f=1.12 p=0.083$, Fig.

228 4c), which is consistent with the formation of meconium prior to birth.

230 Increasing illness severity closely associates with decreasing gestational age and is therefore a

231 covariate and potential confounder in many diseases related to preterm birth ${ }^{18}$. To gauge a potential

232 confounding influence of illness severity, we examined fungal community composition in relation to a

233 critical score of 11 on the CRIB-II. No differences were appreciated in alpha diversity as quantified by

234 the Chao1 index (Supplemental Fig. 4j). The relative abundance differences were also less markedly

235 altered than for other perinatal factors (Supplemental Fig. 4k). The abundance of Saccharomycetales

236 was unaltered at the class level, but the abundance of Polyporales was reduced (15.5\% with score

$237<11$ and $6.9 \%$ with a score $\geq 11$ ) and the relative abundance of Capnodiales and Dothideales

238 increased with elevated CRIB-II score $(6.0 \%<11$ and $13.5 \%, \geq 11$; and $1.6 \%<11$ and $10.8 \% \geq 11$,

239 respectfully). Clinical illness severity did not appear to alter fungal community composition

240 (PERMANOVA $R^{2}=0.0251 p=0.146$ and RDA, variance $=2.68 f=0.85 p=0.947$, Fig. $4 d$ ). 


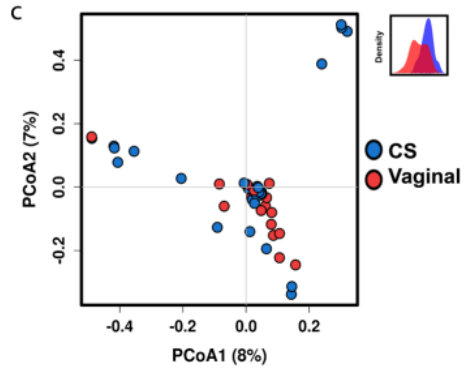

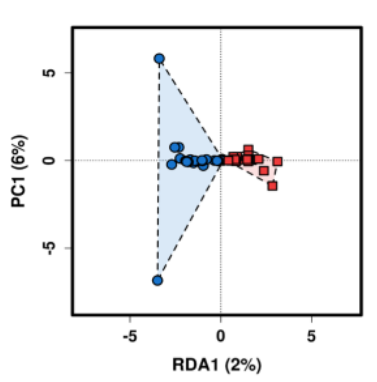

Figure 4

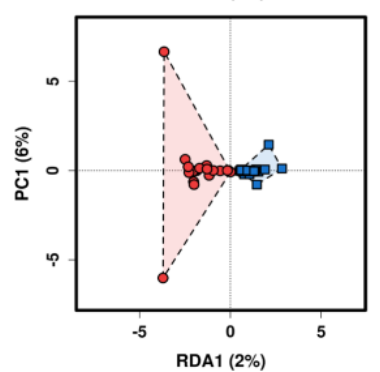

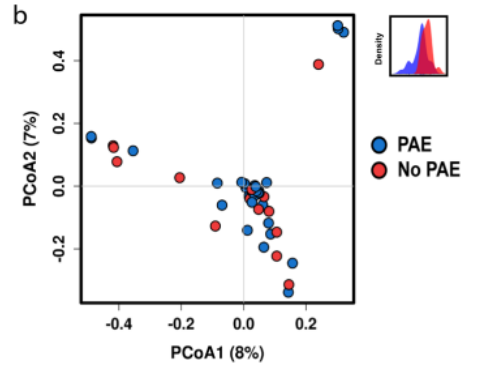
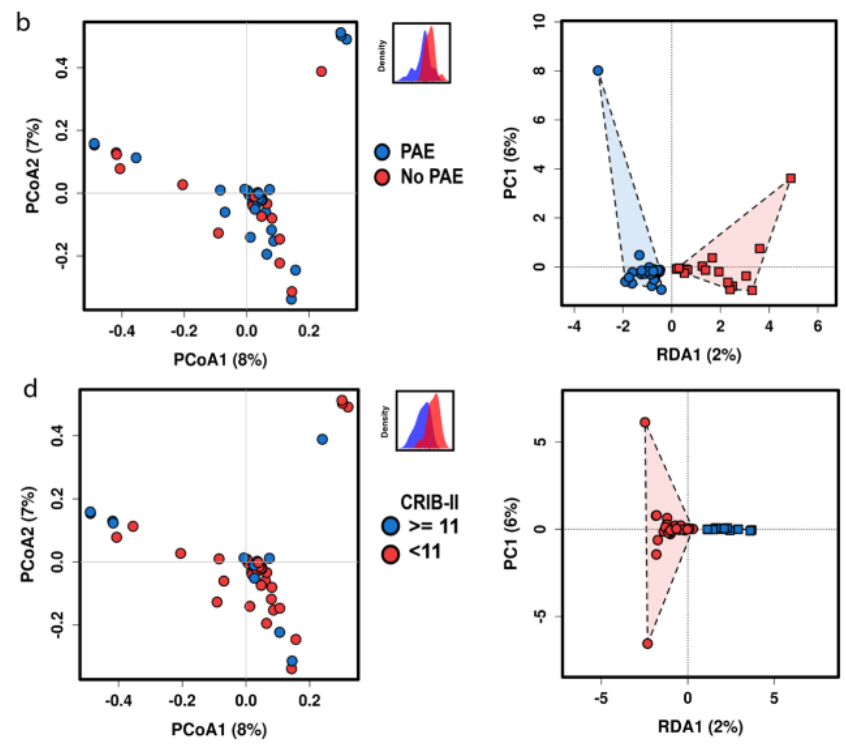

Fig. 4. Fungal community structure is not determined by common perinatal factors.

a Host sex does not significantly alter fungal community composition. Principal coordinates analysis (PCoA) of Bray-Curtis dissimilarity matrices, PERMANOVA $R^{2}=0.0209 p=0.529$. Redundancy analysis (RDA), variance $=3.19 f=1.02 p=0.348$. The subset displays a discriminant analysis of principal components.

b Prenatal antibiotic exposure (PAE) does not significantly alter fungal community composition. PCoA of Bray-Curtis dissimilarity matrices, PERMANOVA $R^{2}=0.202 p=0.598$. RDA, variance $=3.51 f=1.12$ $p=0.083$.

c Mode of delivery does not significantly alter fungal community composition. PCoA of Bray-Curtis dissimilarity matrices, PERMANOVA $R^{2}=0.202 p=0.598$. RDA, variance $=3.51 f=1.12 p=0.083$. d Illness severity does not significantly alter fungal community composition. PCoA of Bray-Curtis dissimilarity matrices, PERMANOVA $R^{2}=0.0251 p=0.146$. RDA, variance $=2.68 f=0.85 p=0.947$. CRIB-II, Clinical Risk Index for Babies II.

For all analyses $n=71$.

\section{Fungi form complex interkingdom communities}

We examined how fungi formed interkingdom microbial communities by the characterizing the resulting community composition from the combined 16 s and ITS sequencing results $(n=71)$. For most infants, fungal communities were a minor component their interkingdom microbiome, but a few preterm infants had microbiomes that were dominated by fungi (Fig. 5 and Supplemental Fig. 1). This may emphasize the importance of pioneer species to the ecological succession of the primordial gut. In general, the interkingdom microbiome increased in complexity with increasing gestational age at birth. While rarefaction analysis favored more microbial richness in term samples (Fig. 5a), alpha diversity as quantified by the Shannon diversity index was not significantly altered (ANOVA $f=2.2 p$ 
$289=0.14$, Fig 5b). PCoA of the combined microbial communities showed clear differential clustering for 290 with a binary cut off at 33 weeks' gestation (Bray-Curtis dissimilarity matrices, PERMANOVA with 999 291 permutations $R^{2}=0.103 p=0.000333$, Fig. 5c). Discriminant analysis of principal components 292 suggests that the principal variables at the genus level are the prevalence of Methylobacterium, 293 Bacteroides and Stereum. The most prominent differences in abundance at the phylum level were in 294 Blasidiomycota, Bacteroidetes and Actinobacteria, all of which were more abundant in term born 295 infants. Acidobacteria, in contrast, were more prevalent in preterm infants (Supplemental Fig. 1b). At 296 the genus level, there were expansions in multiple genera with increasing gestational age. The most 297 prominent changes were in Methylobacterium and Bacteroides (Supplemental Fig. 1d). However, 298 there were some genera, in particular Lactobacillus and Staphylococcus, that were more abundant in 299 preterm infants, but at a much lower level. The expansion of the interkingdom microbiome with 300 increasing gestation is also supported by analysis of the core microbiome of term infants which 301 increased from 24 to 36 unique OTUs and added 7 unique phyla to the core preterm microbiome 302 (Supplemental Fig. 1e).

304 To understand interrelatedness in interkingdom ecology, we used network analysis. Compared to the 305 sparse inter-fungal networks, fungi and bacteria form complex interkingdom networks with numerous 306 interactions (Figure 5d). The expanding networks led to multiple unique nodes clearly separated by 307 gestational age at birth.

309 To characterize the influence of perinatal factors on interkingdom community structure, we used 310 PCoA and RDA to capture the impact of host sex, prenatal antibiotic exposure, mode of delivery and 311 illness severity. Similar to fungal community structure, the interkingdom structure was not significantly 312 altered by these common perinatal exposures as assessed by both constrained and unconstrained 313 analyses (Supplemental Fig. 2). 
a

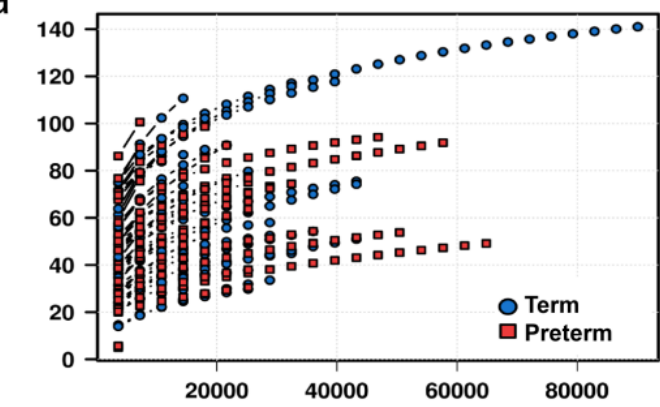

b

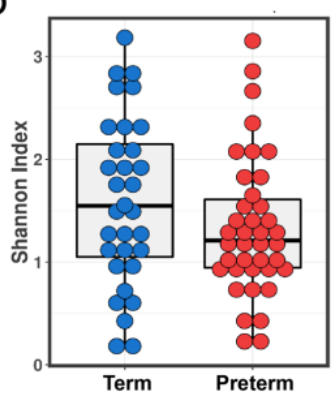

C

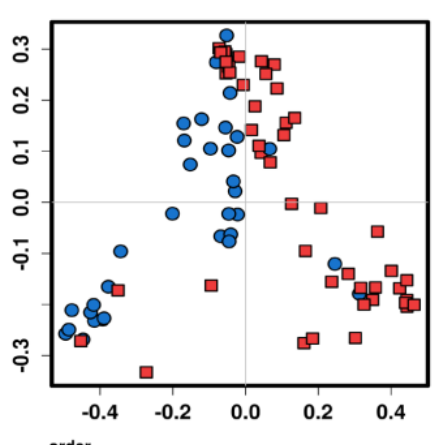

order

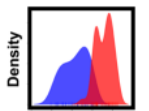

OTerm 口Preterm

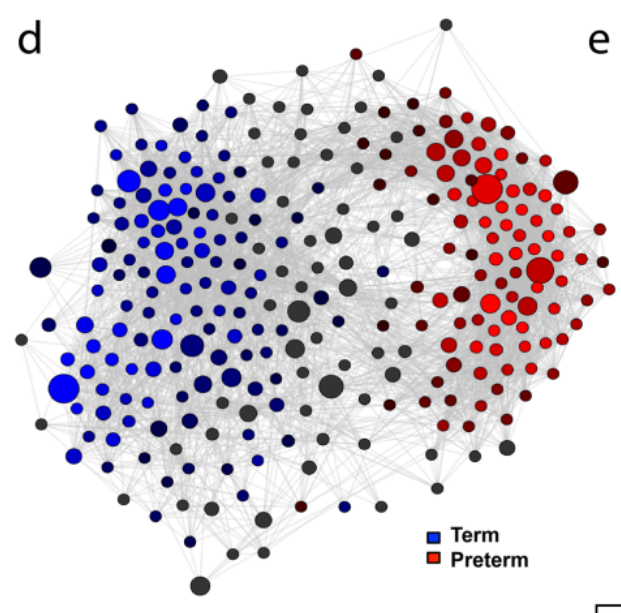

e
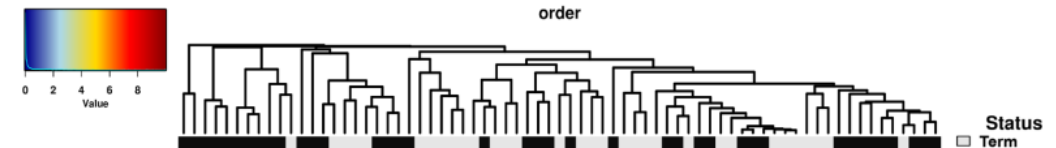
I I I 1

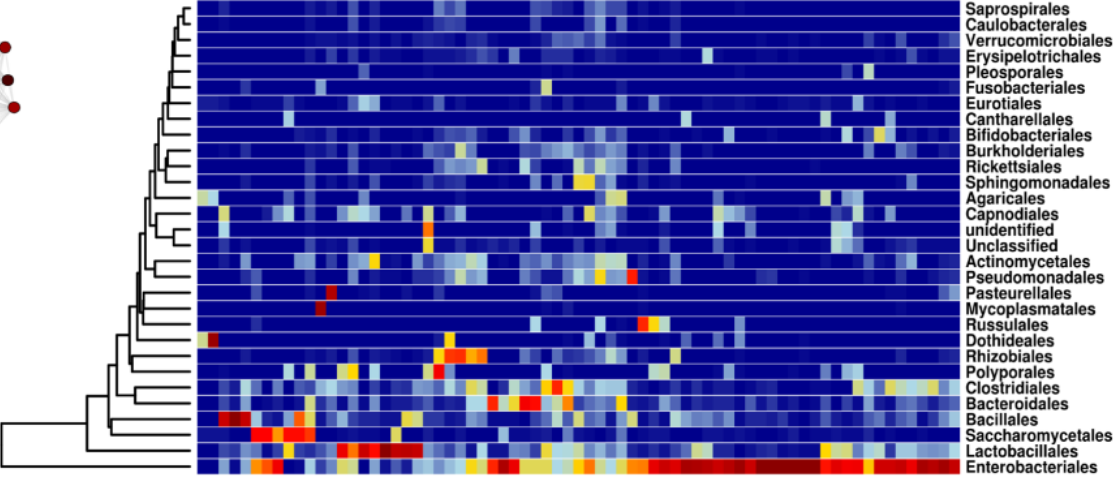

$f$

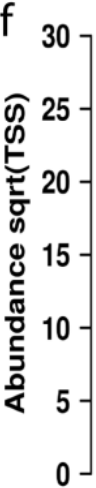

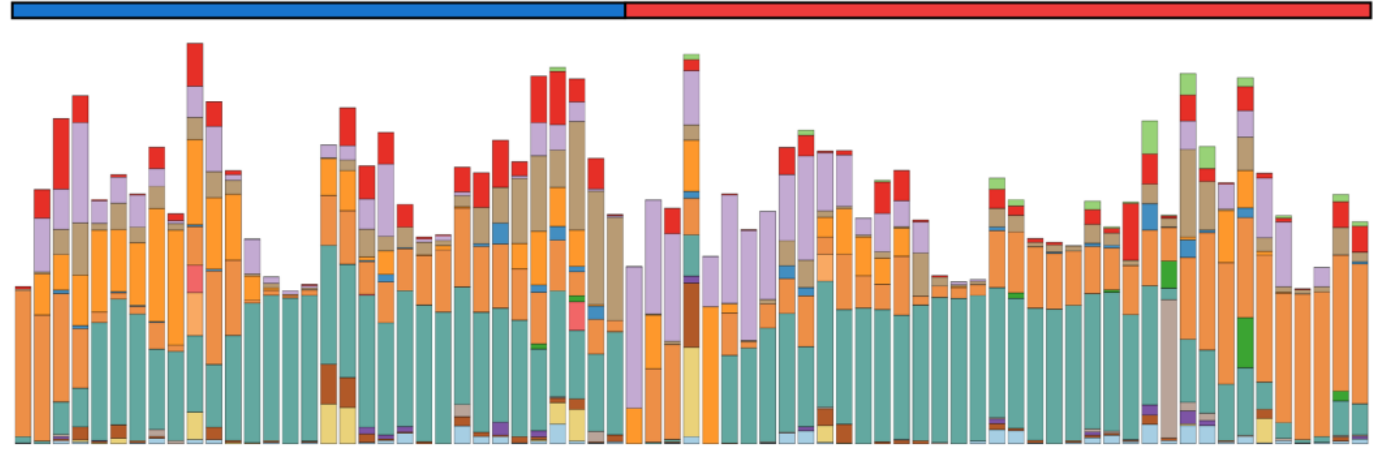

$\square$ Term

Preterm

Features:

- Actinobacteria

Ascomycota

Basteroidetes

Cyanobacteria

$\checkmark$ Firmicutes

․ Fusobacteria

Mortierellomycota

Mucoromycota

$\square$ Tenericutes

Thermi

U Unclassified

unidentified

$\checkmark$ Verrucomicrobia

Fig 5. Fungi and bacteria form complex interkingdom microbial communities in human meconium. a Sequencing rarefaction analysis of species richness.

b Alpha diversity quantified by the Shannon index, ANOVA $f=2.2 p=0.14$.

c Principal coordinates analysis of Bray-Curtis dissimilarity matrices, PERMANOVA with 999 permutations $R^{2}=0.103 p=0.000333$. The subset displays a discriminant analysis of principal components.

d Network analysis developed using Spearman's correlations. Positive correlations with false discovery rate-adjusted, $p$-values $<0.05$ are presented as an edge.

e Heat map of differential distribution of taxa at the order level, ranked by gestation.

f Relative abundance of taxa at the phylum level. Sqrt(TSS), square root total sum normalization

For all analyses $(n=71)$. Preterm samples are displayed in red and term-born samples in blue. 
333

334

335

336

337

\section{Key interkingdom community members predict gestational age of the host}

To explore the distribution of microbial taxa with respect to gestational age at birth, we developed a machine learning model to predict the gestational age of a newborn based on the interkingdom composition of the microbiome. We developed our first machine learning model using only microbial taxa. Starting with 51 fungal and 209 bacterial taxa that were identified at least twice in the dataset, we utilized LASSO with 5 -fold cross validation to identify potential significant predictors, leading to the identification of 9 fungal and 34 bacterial taxa clearly distributed by gestational age (Supplemental Fig. 6a). A support vector machine model reliably classified infants as preterm or term utilizing these taxa (Supplemental Fig. 6b).

\section{Interkingdom community composition classifies preterm status}

To understand the relative strength of key microbial taxa to function as predictors of preterm status relative to other clinical demographic data, we implemented a random forest model with 5-fold cross validation using the presence of 260 genus-level microbial taxa and select clinical demographics. After 100 independent permutations, average sensitivity analysis of the final random forest model provided an area under the curve (AUC) of 0.859 [95\% confidence interval $(\mathrm{Cl})$ 0.857-0.862, Supplemental Fig. 6c]. We then performed a variable importance analysis on the random forest model and selected the top 10 variables to build a rarified random forest model using 5 -fold cross validation. After 100 permutations, the average performance of the sensitivity analysis of the rarified random forest models had an AUC 0.867 (95\% Cl 0.861-0.871). Overall, the strongest predictors from these key taxa ran counter to the general trend and were more abundant in preterm than in term-born infants (Undefined genera within family Rikenellaceae, and the bacterial genera Parabacteroides and Citrobacter). With the exception of Lactococcus, the other key genera were more abundant in termborn infants. Of note, maternal body mass index and age also functioned as reliable predictors, which may be related to their known association with preterm birth ${ }^{19}$. 
363 We compared the random forest models to a variety of other classifiers. All other methods compared

364 provided classification accuracy $<71 \%$, which was significantly lower than the accuracy of the random

365 forest classifiers (Supplemental Fig. 6d). During model development, we did not include clinical

366 variables such as prenatal steroid use, duration of rupture of membranes, antibiotic use and delivery

367 mode as predictors because such variables are closely associated with impending delivery and would

368 confound predictive modeling. However, we implemented Mann-Whitney-U (or Kruskal-Wallis) tests to

369 determine whether there were significant differences between the presence of these variables and the

37010 key microbial taxa we previously identified during development of the random forest model. We

371 found no significant differences between antibiotics use, duration of membrane rupture or delivery

372 mode and presence of these 10 taxa. In contrast, there were statistically significant differences ( $p$

$373<0.01$ ) between use of prenatal steroids (none, 1 or 2 doses) and the abundance of 7 of these key

374 taxa (Supplemental Fig. 7a).

375

376 To quantify the performance of higher-order microbial taxa, we developed models exploring the

377 strength of different phylogenic levels to function as predictors of prematurity. We designed our

378 modeling approach in six levels to capture the influence of using increasingly more granular taxa

379 information on the classification accuracy. The accuracy of the model increased as the as the

380 phylogenic level of the taxa used decreased, highlighting the importance of the distribution of key tax

381 and their variation with gestational age (Fig. 5 and Supplemental Fig. 7b). In addition, when using

382 higher level microbial taxa as potential predictors, the mothers' age and body mass index were

383 relatively more important as predictors. These statistical models demonstrate the differential variation

384 of the interkingdom community composition and underscores the close relationship between the

385 microbial community composition and gestational age of the host at birth. 


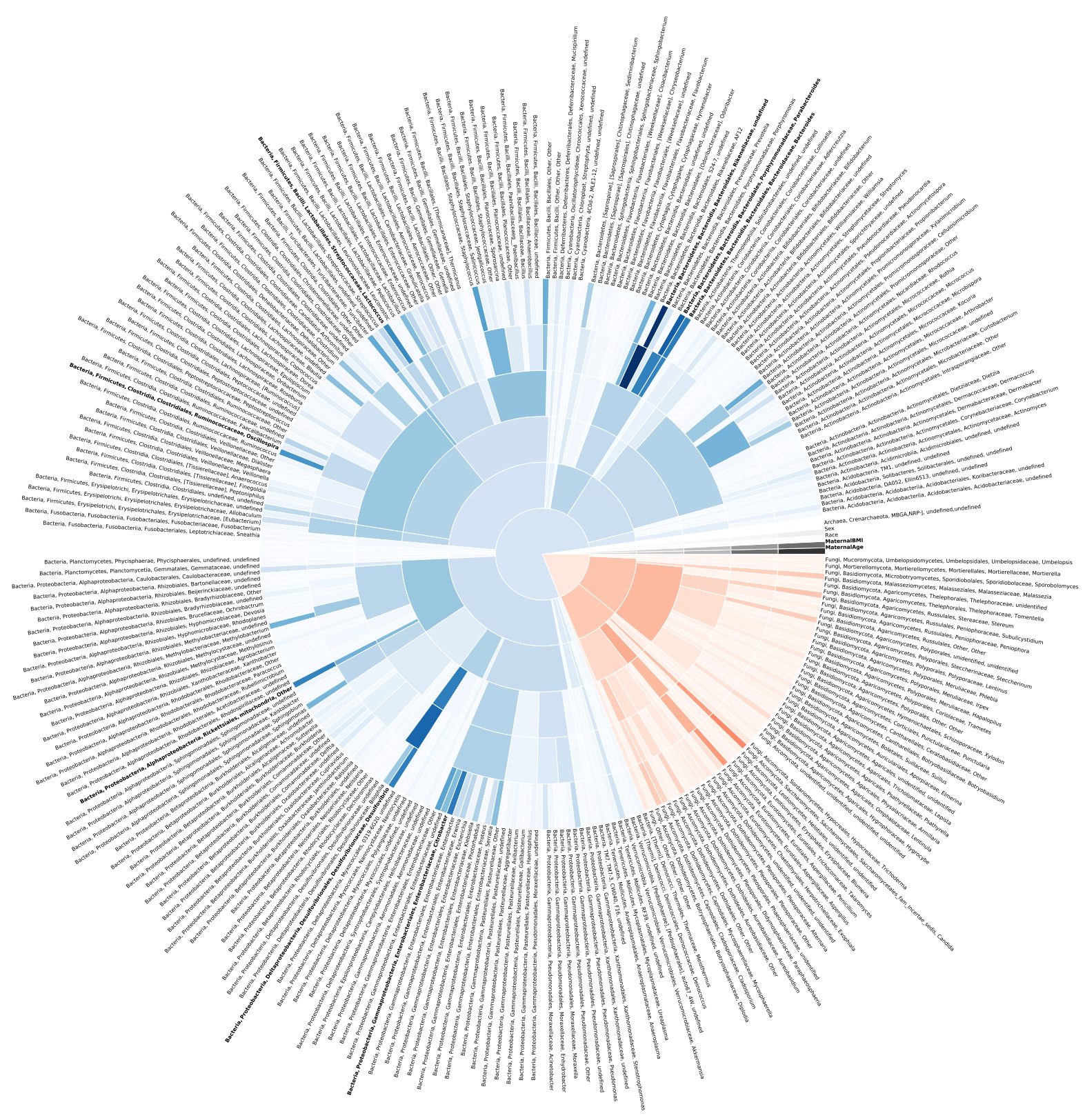

Fig. 6. Interkingdom microbial community composition accurately classifies preterm infants. The precision of rarified random forest classifier machine learning models developed using microbial taxa from each level of the phylogenic tree increases as the phylogenic level decreases, highlighting the importance of key microbial taxa. Darker shading indicates a more important predictor to the machine learning model. Blue represents bacteria, red represents fungi and black represents archaea and clinical demographics. Starting from the center and moving outward, concentric rings represent models developed utilizing taxa on the kingdom, phylum, class, order, family, and genus levels. The 10 most important predictors based on the genus-level model are highlighted in bold. Area under the curve statistics for each level are reported in Supplementary Fig. 6b. 
407

408

409

\section{Fungal and microbial organisms can be cultured from the primordial microbiome}

To explore if our next generation sequencing-based analyses were potentially representative of live microorganisms as opposed to translocated microbial DNA, we performed anaerobic and aerobic culture-based assays for both fungi and bacteria on a subset of samples $(n=41)$ for which sufficient meconium was available (Supplemental Fig. 8). Aerobic fungi-selective culture media was positive in $32 \%$ of preterm and $29 \%$ of term infant samples $\left(\chi^{2} p=0.835\right)$. Aerobic bacteria-selective culture media was positive in $89 \%$ of preterm and $71 \%$ of term infant samples $\left(\chi^{2} p=0.128\right)$. Anaerobic fungiselective culture media was positive in $50 \%$ of preterm and $21 \%$ of term-born infant samples $\left(\chi^{2} p=\right.$ 0.079). Anaerobic bacteria-selective culture media was positive in $69 \%$ of preterm and $88 \%$ of term infant samples $\left(\chi^{2} p=0.1543\right)$. Overall, bacterial cultures were diffusely positive, while fungal cultures were less likely to demonstrate growth. While contamination cannot be completely excluded, we also cannot eliminate the possibility of live fungi and bacteria in the primordial human gut.

\section{The fetal gut contains fungal DNA}

Considerable controversy persists as to whether the isolation of microbial DNA from the human placenta or newborn gut is representative of live microbial organisms ${ }^{20}$. While the similarities between microbes observed in the placenta and neonatal meconium suggest transfer of microbial DNA across the placenta, they do not represent direct observation of the fetal intestine. However, bacterial DNA has been identified in the fetal gut in a mouse model of normal pregnancy ${ }^{21}$. To directly investigate the fetal intestine for the presence of fungal DNA and live organisms we developed a mouse model of normal pregnancy. Using C57BL6J mice, we collected samples from the fetal gut and several maternal body sites on either estimated gestational day 17 (E17) or within $24 \mathrm{~h}$ of birth (P1). We used real time PCR to identify ITS biomass across all maternal and fetal samples. The relative fungal biomass was about 14 -fold lower in the fetal intestine before birth than in the maternal amniotic fluid. However, fungal biomass was about 6-fold more abundant in the neonatal intestine than the amniotic 
435 fluid (mean cycle threshold, $C_{t}=36.75 \pm 15.48$ at $E 17$ and $C_{t}=25.71 \pm 15.16$ at $P 1$ and $C_{t}=29.20$

$436 \pm 6.58$ in amniotic fluid).

437

438 Dectin-1 is a pattern recognition receptor that recognizes the fungal cell-wall $\beta$-glucan and is important

439 for detection of fungal pathogens in humans and mice ${ }^{22}$. To investigate if inhibited host sensing of

440 fungi altered the transplacental deposition of fungal biomass in the neonatal gut, we collected

441 samples from the fetal gut and multiple maternal body sites from dectin-1/- mice on the C57BI6J

442 background (B6.clec7a ${ }^{\text {tm1Gdb }}$ ). In the fetal intestine, dectin-1/- mice had about a 6-fold increase in

443 fungal biomass over dectin- 1 competent mice $\left(C_{t}=33.50 \pm 14.81\right.$ versus $\left.C_{t}=36.75 \pm 15.16\right)$.

444 However, after birth, the neonatal intestinal fungal biomass was lower on $P 1\left(C_{t}=29.33 \pm 18.01\right.$

445 versus $C_{t}=25.71 \pm 15.48$ ) and remained a more significant proportion of the intestinal biomass. While

446 there are considerable differences between the placentas of humans and mice ${ }^{23}$, these results

447 suggest that the intrauterine transfer of fungal DNA may be widespread across mammals. Impaired

448 host-sensing of fungi may alter both intrauterine deposition and post-natal colonization.

Here we present the first description of the primordial fungal ecology of the neonatal gut using both

454 next-generation sequencing and culture-based techniques. Microeukaryotes, primarily fungi, were

455 present in the first-pass meconium samples collected within hours of birth in both preterm and term-

456 born newborns. In general, as quantified by amplicon sequencing of eukaryotic ITS rDNA, infantile

457 fungal communities developed greater taxonomic diversity as the gestational age at birth increased.

458 The structure of these communities was unaffected by perinatal factors, such as mode of delivery,

459 antibiotic exposure, infant sex, and illness severity, consistent with intrauterine formation of

460 meconium. Culture-based techniques indicate microbial DNA may originate from viable

461 microorganisms present in the neonatal gut. 
463 Understanding the primary succession of microorganisms into the human gut is important because

464 the order in which these potential colonizers are introduced into the intestine influences the mature

465 community structure. Colonization experiments in mice suggest the timing of arrival of microbial

466 colonizers is the major factor in determining final community structure ${ }^{24}$; which helps explain why

467 other perinatal factors, such as perinatal antibiotic exposure, account for only limited variability in

468 mature community structure. Bacterial communities then undergo a rapid expansion during the first

469 several years of life ${ }^{25}$, eventually establishing a host-specific core microbiome that resists further

470 perturbations ${ }^{26}$. Because misconfiguration of the core microbiome during this period likely contributes

471 to multiple host disease states, understanding the ecological processes driving microbiome

472 assemblage is crucial to developing therapeutic interventions ${ }^{27}$. Recent evidence suggests that the

473 mycobiome remains relatively constant during the first year of life $e^{11,12}$, which underscores that the

474 primary succession of initial fungal colonizers may be even more important for final community

475 structure than the succession of bacterial species. In addition, fungi may also form a crucial nidus for

476 bacterial colonization ${ }^{6}$. Our evidence for the presence of commensal fungi in the primordial gut and

477 the development of these communities with gestational age demonstrates this process may begin

478 earlier that was previously thought - with implications that extend throughout life.

480 Meconium forms during early human gut development, eventually migrating into the colon around the $48119^{\text {th }}$ week of gestation ${ }^{28}$. Passage of meconium is a developmentally programmed event that usually 482 occurs within 48 hours after birth ${ }^{28}$. Once enteral feeding has been established environmental 483 microbes invade the gastrointestinal tract and are detectable in the first true stools. Until the 484 development of next-generation sequencing techniques, the presence of microorganisms in the first485 pass meconium was opaque using traditional culture-based techniques. Meconium, and therefore the 486 uterine environment, has been widely accepted to be sterile ${ }^{9}$. However, during the past decade 487 several researchers have demonstrated bacterial DNA can be isolated from meconium ${ }^{3,8,29-34}$ and the 488 placenta ${ }^{35-37}$, but if this microbial biomass is representative of live organisms remains controversial. 
489 These studies have focused on the bacterial components of meconium, however, leaving fungi 490 undescribed.

492 The human amniotic fluid may also harbor a very low level of microbial organisms. Chu et al. ${ }^{17}$ have 493 demonstrated that the bacterial components of the meconium microbiome are distinct and do not 494 resemble adult vaginal and skin communities like the predominant bacterial members of other 495 neonatal body sites. Our results show interkingdom microbial communities that are essentially 496 unaffected by perinatal exposures, with the possible exception of prenatal steroids. The 497 demonstration of live microorganisms in the newborn gut represents a monumental shift from the 498 sterile womb hypothesis that dominated perinatal biology for the last century ${ }^{8,9}$. The increasing 499 prevalence and the development of bacterial ecology with increasing gestational age suggests that 500 this is a natural, non-pathogenic phenomenon ${ }^{3,33,34}$. The human fetus regularly swallows and 501 replenishes the amniotic fluid throughout gestation, which suggests a mechanism for how rare 502 microbes in the amniotic fluid could become more concentrated within the primordial gut. Based on 503 these findings, we postulate that low abundant amniotic fluid bacterial and fungal biomass is 504 continuously collected during gestation and accumulates in the intestine. By the time of delivery, 505 meconium may contain a collective record of fetal microbial exposure in an analogous manner to how 506 meconium contains a record of transplacental illicit drug exposure ${ }^{38}$. This process would also expose 507 the fetal mucosal immune system to low levels of microbial products that may initiate host microbial 508 surveillance mechanisms required to process the influx of microbes into the gut that occurs during and 509 after delivery.

511 To directly observe the fetal environment, we utilized a mouse model of normal pregnancy. This work 512 highlights the presence of fungal biomass within the developing fetal gut. The implication of a steady 513 deposition of microbial biomass in the fetal intestine suggests that this is a natural process, which 514 raises the question of whether translocation of microbes is regulated. To gain insight into this process, 
515 we asked if impairment of the host's recognition of fungi would alter the deposition of fungal biomass

516 within the fetal and neonatal gut. Indeed, we found that loss of the host fungal pattern recognition

517 receptor Dectin-1 resulted in more fungal biomass within the fetal intestine. While there are marked

518 differences between the placentas of humans and mice ${ }^{21}$, this suggests that the deposition of fungal

519 biomass within the fetal gut may be a regulated process that requires host recognition of fungal

520 organisms.

521

522 Counter to the general trend of our results which support an apparently natural process of microbial

523 biomass accumulation in the neonatal intestine, our finding that some preterm infants are dominated

524 by fungal order Saccharomyces, and in particular the genus Candida, may support the alternate

525 hypothesis that in some instances preterm birth is a product of polymicrobial disease of the uterine

526 cavity $^{39}$. After birth, systemic infection with Candida are a persistent threat during neonatal care.

527 Candida species, primarily Candida albicans, are opportunistic pathogens particularly associated with

528 bacterial dysbiosis ${ }^{40}$. Candida are significant commensals of the human gut ${ }^{41}$, so the presence of

529 Candia within the gastrointestinal tract is understandable. In the mature gut, commensalism is

530 maintained by a tripartite interaction between Candida, other members of the microbiome, and host

531 immunity ${ }^{41}$. However, Candida are also the dominate fungal species associated with microbial

532 invasion of the amniotic cavity ${ }^{42}$, and are detectable in the amniotic fluid by culture and culture-

533 independent techniques ${ }^{43}$. As quantified by culture-based techniques, Candida chorioamnionitis is a

534 rare but accepted cause of preterm birth ${ }^{44}$. Our study showed that preterm meconium samples were

535 more likely to contain fungi than term infants. Furthermore, the mycobiomes of preterm infants were

536 also more likely to be dominated by Candida, as opposed to the more diverse mycobiomes of infants

537 with a longer gestation. Regardless of these concerning findings, conclusively demonstrating that

538 Candida colonization is associated with preterm birth will require a clinical trial powered to test this

539 hypothesis. 
541 Fungi are ubiquitous in the environment. In low biomass samples like meconium, the presences of

542 environmental fungi are difficult to separate from organisms originating within the samples. Because

543 microeukaryotic DNA is a relatively low proportion of the fecal DNA content, especially in meconium,

544 exacting extraction, purification and amplification techniques are required. A prominent reason that

545 investigation of the mycobiome has lagged behind investigation of the microbiome is a lack of

546 standardization in detection methods for fungal organisms ${ }^{41}$. Our choice to utilize amplification of the

547 ITS2 region, the read depth and the database used to align our amplicon reads, therefore, could all

548 influence the fungal species identified in our samples. Incidentally, the exacting DNA extraction

549 techniques we utilized may also explain the differences in bacterial community structure with prior

550 work because they increased the detectability of low biomass samples. We froze meconium samples

551 immediately after collection to prevent post-excretion microbial expansion that would alter the relative

552 composition of microbial communities, and passed negative control samples throughout the sample

553 collection, DNA extraction and amplicon sequencing process. However, the fact that perinatal

554 exposures known to shape the post-natal microbiome did not significantly alter community

555 composition in our samples argues that significant post-natal microbial colonization did not occur

556 before sample collection.

558 Our findings support the presence of fungal organisms within the neonatal gut. Taken together, our

559 sequencing and culture-based techniques support the presence of live fungal organisms in meconium

560 in addition to bacteria. Homeostasis of fungal commensals may represent an important aspect of

561 human biology present even before birth with functional implications throughout early life. Low

562 abundance colonization of the primordial gut with interkingdom ecologies appears to be a normal part

563 of healthy pregnancy that might represent a priming of the intestine for the exponential expansion of

564 the microbiome after birth. The domination of microbial communities by fungal order Saccromycetes

565 in some preterm infants, however, suggests a potential pathologic association with preterm birth. 
568

569

570

571

572

573

574

575

576

577

578

579

580

581

582

583

584

585

586

587

588

589

590

591

592

593

594

595

596

597

598

599

600

\section{Methods}

\section{Study patients}

Written and informed consent was obtained from the infants' mothers. All investigations were carried out in accordance with the declaration of Helsinki under The University of Tennessee Health Science Center Institutional Review Board protocol 17-05311-XP. We prospectively enrolled either very low birthweight infants $(<1500 \mathrm{~g})$ preterm or term-born (estimated gestational age 37-41 weeks) infants (Fig. 1a). We excluded any mother-infant dyads with the potential for immune deficiency, but otherwise invited all mothers that meet our entrance criteria during the study period to participate.

\section{Infant exposures}

Data was collected regarding infant demographics and the following clinical exposures: prenatal and postnatal antibiotic exposure, perinatal steroid exposure, delivery mode, and illness severity (the CRIB-II ${ }^{18}$. Additional clinical characteristics were also abstracted from the medical record to characterize potential co-variates with gestational age.

\section{Sample collection}

The first-pass meconium was collected during the first $48 \mathrm{~h}$ after birth. Samples were collected from diapers using sterile spatulas, placed into sterile microcentrifuge tubes and frozen at $-30 \mathrm{C}$ within minutes of collection. Several times a week, batches of samples were collected and frozen at $-80 \mathrm{C}$ until further analysis could be performed. Infants that did not stool during the first $48 \mathrm{~h}$ or for which a meconium sample was not collected were excluded from downstream analysis.

\section{DNA extraction and Illumina MiSeq sequencing}

Meconium samples were resuspended in $500 \mu \mathrm{L}$ of TNES buffer containing 200 units of lyticase and $100 \mu \mathrm{L}$ of $0.1 / 0.5$ (50/50 Vol.) zirconia beads. Incubation was performed for $20 \mathrm{~min}$ at $37^{\circ} \mathrm{C}$. Following mechanical disruption using ultra-high-speed bead beating, $20 \mu \mathrm{g}$ of proteinase $\mathrm{K}$ was added to all samples, and they were incubated overnight at $55 \mathrm{C}$ with agitation. Total DNA was extracted using 
601 chloroform isoamyl alcohol, and total DNA concentration per mg stool was determined by qRT-PCR.

602 Purified DNA samples were sent to the Argonne National Laboratory (Lemont, IL) for amplicon

603 sequencing using the NextGen Illumina MiSeq platform. Blank samples passed through the entire

604 collection, extraction and amplification process remained free of DNA amplification.

605

606

607

608 Sequencing data were processed and analyzed using QIIME (Quantitative Insights into Microbial

609 Ecology) 1.9.1. Sequences were first demultiplexed, then denoised and clustered into sequence

610 variants. For bacteria we rarified to a depth of 3,000 sequences. (In combined analysis after excluding

611 samples with a bacterial depth of $<3,000$, we used a depth of 300 for further analysis).

612 Representative bacterial sequences were aligned via PyNAST, taxonomy assigned using the RDP

613 Classifier. Processed data was then imported into Calypso 8.84 for further analysis and data

614 visualization ${ }^{45}$. The Shannon Index was used to quantify alpha diversity (inter-sample) ${ }^{46,47}$.

615 Unweighted UniFrac analysis was used to quantify beta diversity (intra-sample) ${ }^{48}$, and the differences were compared using PERMANOVA with 999 permutations. For fungi, sequences were aligned, and

617 taxonomy was assigned using the UNITE (dynamic setting) database ${ }^{49}$. Fungal OTUs were rarified at

618 a depth of 300 sequences for alpha diversity using Shannon or Chao1 and beta diversity using Bray-

619 Curtis dissimilarity or Jaccard abundance ${ }^{50}$ indices. As with bacteria, beta diversity significance was

620 then assessed using PERMANOVA. To quantify relative abundance of taxa between groups, we

621 utilized ANOVA adjusted using the Bonferroni correction and FDR for multiple comparisons. We used

622 LEfSe to test for significance and perform high-dimensional biomarker identification ${ }^{51}$. Network

623 analysis was generated from Spearman's correlations. Positive correlations with an FDR-adjusted, $p$

$624<0.05$ were presented as an edge.

625

626

627

628

629

630

631 


\section{Machine learning and variable selection}

633

634

In the first phase, we developed a support vector machine utilizing 34 bacterial and 9 fungal taxa identified utilizing an unbiassed LASSO approach with 5-fold cross validation and generated a confusion matrix to characterize the performance of the model as a predictor of gestational age

In the second phase, we developed a random forest-based classification model to distinguish between preterm and term-born newborns using maternal age, maternal body mass index, newborn sex and 260 microbial taxa that were present in at least two samples in the data set. Random forest classifiers are based on the arrangement of multiple decision trees built using a random sample of predictors. The outcome of a random forest is based on a weighted average of the outcomes of individual decision trees within the model. To identify and control for potential overfitting, we implemented a 5-fold cross validation strategy. By repeating this process 5 times for each subject, we obtained a single class prediction that is purely based on a classifier built on a distinct dataset. We then repeated the development of the entire model 100 times. We also implemented a recursive variable selection based on the out-of-bag predictor importance analysis. Here, variable importance was assessed by randomly permitting values of a single predictor and quantifying the increment in the out-of-bag error rate. Variables that caused no drop on the error rate were assumed to have no influence in predicting outcome. We then selected the 10 most important variables and built another 100 permutations of our random forest model using 5 -fold cross validation.

We also compared the performance of the random forest classifier we generated using this method to other classifiers including logistic regression, discriminant analysis, k-nearest neighborhood, decision trees, support vector machines and neural networks using AUC statistics. Finally, we calculated commonly used classification accuracy statistics such as specificity, sensitivity, and positive predictive value for the final random forest model to characterize the ability of the model to distinguish between preterm and term-born babies. 
659 In the final phase, we repeated this model development process for each phylogenic level to

660 understand the variable importance of different taxa. To achieve this, in level 1 (kingdom) we used

661 four demographic variables and 3 microbial kingdoms (bacteria, fungi, and archaea) features by

662 aggregating all bacterial and fungal taxa under their particular kingdom definition. Therefore, at level 1

663 (kingdom), we built a random forest classifier using a total of 6 features. The four demographics

664 variables included host sex (female: 0 , male: 1 ), maternal self-reported race/ethnicity (Black: 0 , other:

665 1), maternal age, and maternal BMI. Fungal and bacterial taxa were represented in six different levels

666 following, kingdom $>$ phylum $>$ class $>$ order $>$ family $>$ genus. For level 2 (phylum) we aggregated

667 taxa data at 23 distinct phylum-level taxa resulting in total of 27 features and including the same four

668 demographics data. We repeated this process until we had reached the $6^{\text {th }}$ level (genus) where we

669 utilized a total of 265 variables (4 demographics and 261 taxa data at genus level). At each level, we

670 included only taxa that were observed in at least two different subjects to avoid overfitting. For each

671 level, we implemented five-fold cross validation. To avoid sampling bias, we repeated the cross-

672 validation after randomly shuffling the data for 100 times at each level. Classification performance was

673 summarized as AUC statistics.

674

675

676

677

\section{Microbial culture}

We prepared culture broth using either brain heart infusion (BHI) with $250 \mathrm{mg} / \mathrm{L}$ of fluconazole (bacteria-selective) or yeast-extract-peptone-dextrose (YPD) with $50 \mathrm{mg} / \mathrm{mL}$ of chloramphenicol (fungal-selective) under both aerobic and anaerobic conditions. Meconium samples were thawed and diluted in $1 \mathrm{~mL}$ of PBS. Tubes containing $1 \mathrm{~mL}$ of culture broth were inoculated with $100 \mu \mathrm{L}$ of room temperature meconium diluted in $1 \mathrm{~mL}$ of culture broth. Samples were then incubated at $32^{\circ} \mathrm{C}$ under either aerobic or anaerobic conditions. Bacterial samples were assessed by optical density after $48 \mathrm{~h}$ and fungal samples after $120 \mathrm{~h}$. 
689

690

691

692

693

694

695

696

697

698

699

700

701

702

703

704

705

706

707

708

709

710

711

712

713

714

715

716

717

718

719

720

721

722

\section{Statistical analysis}

We compared demographic and clinical variables between infants with a gestational age $<33$ weeks' and $>33$ weeks' using the $\chi^{2}$ or two-tailed Welch $t$-test for parametric variables or the Wilcoxon ranksum test for non-parametric variables, as appropriate.

\section{Animal model of pregnancy}

All animal experiments were approved by the University of Tennessee Health Science Center Institutional Animal Care and Use Committee. Timed-mating of adult C57BL6J mice or dectin-1/ on the C57BI6J background (B6.clec7a ${ }^{\text {tm1Gdb }}$, Jackson Labs stock \#012337) at 8-10 weeks of age was performed to obtain offspring samples at embryonic day 17 (E17) or post-partum day 1 (P1). After euthanasia, swabs were collected from the maternal mouth, skin and genitourinary tract. The dam was then soaked in exspor (chlorine dioxide disinfectant). Gravid uteri and intestines were removed from prepped dams in a UV-light sterilized laminar flow hood by sterile gowned and gloved personnel extensively trained in sterile technique (a maternal-fetal medicine specialist gynecologist and a neonatologist) using sterile technique and instruments that were sterilized between animals. Utilizing a second set of sterilized instruments, pups were removed, euthanized and placed in exspor then passed to another operator who removed them in a second laminar flow hood. The body cavity was opened with another set of sterile instruments and removed the intestine en bloc and placed them into a sterile container. Samples of the placenta, amniotic fluid, small intestine and colonic contents were also placed in sterile containers. All samples were snap frozen and stored at -80C until DNA extraction.

\section{Data Availability}

Microbial sequence data is available at: [To be uploaded on acceptance]

\section{Computer Code}

Custom computer code is available upon request. 


\section{Disclosure}

The authors declare no conflict of interest.

\section{Author Contributions}

$\mathrm{KW}, \mathrm{AT}$ and JFP conceived of the study design; KW and JHP recruited the patients and collected the meconium samples; KW and EM extracted clinical data from the medical record; CG and JFP performed the DNA purification; KW, BP and JFP performed the culture-based analyses; KW and MA performed the murine experiments, IK and OA performed the statistical modeling and developed the machine learning models; $\mathrm{KW}, \mathrm{IK}, \mathrm{OA}$ and JFP performed the data quality control, statistical analysis and prepared the figures; KW wrote the paper; KW, MA, JHP, OA, BP, AT and JFP edited the manuscript; and all authors approved the final version.

\section{Acknowledgements}

The authors would like to thank the mothers and infants who contributed to the study and to our research coordinators, Gail Camp and Nancy Ruch, for their assistance in identifying and recruiting patients.

\section{References}

1. Romano-Keeler, J. \& Weitkamp, J.-H. Maternal influences on fetal microbial colonization and immune development. Pediatr. Res. 77, 189-195 (2015).

2. Mueller, N. T. et al. Delivery Mode and the Transition of Pioneering Gut-Microbiota Structure, Composition and Predicted Metabolic Function. Genes (Basel) 8, 364 (2017).

3. Ardissone, A. N. et al. Meconium Microbiome Analysis Identifies Bacteria Correlated with Premature Birth. PLOS ONE 9, e90784 (2014).

4. Dominguez-Bello, M. G. et al. Delivery mode shapes the acquisition and structure of the initial microbiota across multiple body habitats in newborns. Proc Natl Acad Sci U S A 107, 1197111975 (2010).

5. Olin, A. et al. Stereotypic Immune System Development in Newborn Children. Cell 174, $1277-$ 1292.e14 (2018).

6. Zhang, Y., Kastman, E. K., Guasto, J. S. \& Wolfe, B. E. Fungal networks shape dynamics of bacterial dispersal and community assembly in cheese rind microbiomes. Nat Commun $\mathbf{9}, 336$ (2018).

7. Arrieta, M.-C. et al. Associations between infant fungal and bacterial dysbiosis and childhood atopic wheeze in a nonindustrialized setting. J. Allergy Clin. Immunol. 142, 424-434.e10 (2018). 
763

764

765

766

767

768

769

770

771

772

773

774

775

776

777

778

779

780

781

782

783

784

785

786

787

788

789

790

791

792

793

794

795

796

797

798

799

800

801

802

803

804

805

806

807

808

809

810

811

812

813

8. Jiménez, E. et al. Is meconium from healthy newborns actually sterile? Res. Microbiol. 159, 187-193 (2008).

9. Mackie, R. I., Sghir, A. \& Gaskins, H. R. Developmental microbial ecology of the neonatal gastrointestinal tract. Am. J. Clin. Nutr. 69, 1035S-45S (1999).

10. Limon, J. J., Skalski, J. H. \& Underhill, D. M. Commensal Fungi in Health and Disease. Cell Host Microbe 22, 156-165 (2017).

11. Ward, T. L. et al. Development of the Human Mycobiome over the First Month of Life and across Body Sites. mSystems 3, e00140-17 (2018).

12. Schei, K. et al. Early gut mycobiota and mother-offspring transfer. Microbiome 5, 107 (2017).

13. Chernikova, D. A. et al. The premature infant gut microbiome during the first 6 weeks of life differs based on gestational maturity at birth. Pediatr. Res. 84, 71-79 (2018).

14. Strati, F. et al. Age and Gender Affect the Composition of Fungal Population of the Human Gastrointestinal Tract. Front Microbiol 7, 1227 (2016).

15. Becattini, S., Taur, Y. \& Pamer, E. G. Antibiotic-Induced Changes in the Intestinal Microbiota and Disease. Trends in Molecular Medicine 22, 458-478 (2016).

16. Samonis, G. et al. Prospective evaluation of effects of broad-spectrum antibiotics on gastrointestinal yeast colonization of humans. Antimicrob. Agents Chemother. 37, 51-53 (1993).

17. Chu, D. M. et al. Maturation of the infant microbiome community structure and function across multiple body sites and in relation to mode of delivery. Nat. Med. 23, 314-326 (2017).

18. Parry, G., Tucker, J., Tarnow-Mordi, W.UK Neonatal Staffing Study Collaborative Group. CRIB II: an update of the clinical risk index for babies score. The Lancet 361, 1789-1791 (2003).

19. Kong, L., Nilsson, I. A. K., Gissler, M. \& Lavebratt, C. Associations of Maternal Diabetes and Body Mass Index With Offspring Birth Weight and Prematurity. JAMA Pediatr 173, 371 (2019).

20. Perez-Muñoz, M. E., Arrieta, M.-C., Ramer-Tait, A. E. \& Walter, J. A critical assessment of the "sterile womb" and "in utero colonization" hypotheses: implications for research on the pioneer infant microbiome. Microbiome 5, 243 (2017).

21. Martinez, K. A. et al. Bacterial DNA is present in the fetal intestine and overlaps with that in the placenta in mice. PLOS ONE 13, e0197439 (2018).

22. Schorey, J. S. \& Lawrence, C. The pattern recognition receptor Dectin-1: from fungi to mycobacteria. Curr Drug Targets 9, 123-129 (2008).

23. Schmidt, A., Morales-Prieto, D. M., Pastuschek, J., Fröhlich, K. \& Markert, U. R. Only humans have human placentas: molecular differences between mice and humans. J. Reprod. Immunol. 108, 65-71 (2015).

24. Martínez, I. et al. Experimental evaluation of the importance of colonization history in early-life gut microbiota assembly. Elife 7, 1 (2018).

25. Arrieta, M.-C., Stiemsma, L. T., Amenyogbe, N., Brown, E. M. \& Finlay, B. The Intestinal Microbiome in Early Life: Health and Disease. Front. Immunol. 5, 355 (2014).

26. Martínez, I., Muller, C. E. \& Walter, J. Long-term temporal analysis of the human fecal microbiota revealed a stable core of dominant bacterial species. PLOS ONE 8, e69621 (2013).

27. Koskella, B., Hall, L. J. \& Metcalf, C. J. E. The microbiome beyond the horizon of ecological and evolutionary theory. Nat Ecol Evol 1, 1606-1615 (2017).

28. Ahanya, S. N., Lakshmanan, J., Morgan, B. L. G. \& Ross, M. G. Meconium Passage in Utero: Mechanisms, Consequences, and Management. Obstet Gynecol Surv 60, 45-56 (2005).

29. Rougé, C. et al. Investigation of the intestinal microbiota in preterm infants using different methods. Anaerobe 16, 362-370 (2010).

30. Mshvildadze, M. et al. Intestinal microbial ecology in premature infants assessed with nonculture-based techniques. J Pediatr 156, 20-25 (2010).

31. Gosalbes, M. J. et al. Meconium microbiota types dominated by lactic acid or enteric bacteria are differentially associated with maternal eczema and respiratory problems in infants. Clinical \&amp; Experimental Allergy 43, 198-211 (2013). 
814

815

816

817

818

819

820

821

822

823

824

825

826

827

828

829

830

831

832

833

834

835

836

837

838

839

840

841

842

843

844

845

846

847

848

849

850

851

852

853

854

855

856

857

858

32. Stewart, C. J. et al. The preterm gut microbiota: changes associated with necrotizing enterocolitis and infection. Acta Paediatr 101, 1121-1127 (2012).

33. Hansen, R. et al. First-Pass Meconium Samples from Healthy Term Vaginally-Delivered Neonates: An Analysis of the Microbiota. PLOS ONE 10, e0133320 (2015).

34. Tapiainen, T. et al. Maternal influence on the fetal microbiome in a population-based study of the first-pass meconium. Pediatr. Res. 84, 371-379 (2018).

35. Collado, M. C., Rautava, S., Aakko, J., Isolauri, E. \& Salminen, S. Human gut colonisation may be initiated in utero by distinct microbial communities in the placenta and amniotic fluid. Sci Rep 6, 23129 (2016).

36. Aagaard, K. et al. The placenta harbors a unique microbiome. Sci Trans/ Med 6, 237ra65237ra65 (2014).

37. Zheng, J. et al. The Placental Microbiome Varies in Association with Low Birth Weight in FullTerm Neonates. Nutrients 7, 6924-6937 (2015).

38. Palmer, K. L. \& Krasowski, M. D. Alternate Matrices: Meconium, Cord Tissue, Hair, and Oral Fluid. Methods Mol. Biol. 1872, 191-197 (2019).

39. Payne, M. S. \& Bayatibojakhi, S. Exploring preterm birth as a polymicrobial disease: an overview of the uterine microbiome. Front. Immunol. 5, 595 (2014).

40. Iliev, I. D., Immunology, I. L. N. R.2017. Fungal dysbiosis: immunity and interactions at mucosal barriers. nature.com

41. Neville, B. A., d'Enfert, C. \& Bougnoux, M.-E. Candida albicans commensalism in the gastrointestinal tract. FEMS Yeast Res. 15, fov081 (2015).

42. DiGiulio, D. B. Diversity of microbes in amniotic fluid. Semin Fetal Neonatal Med 17, 2-11 (2012).

43. DiGiulio, D. B. et al. Prevalence and Diversity of Microbes in the Amniotic Fluid, the Fetal Inflammatory Response, and Pregnancy Outcome in Women with Preterm Pre-Labor Rupture of Membranes. American Journal of Reproductive Immunology 64, 38-57 (2010).

44. Maki, Y., Fujisaki, M., Sato, Y. \& Sameshima, H. Candida Chorioamnionitis Leads to Preterm Birth and Adverse Fetal-Neonatal Outcome. Infect Dis Obstet Gynecol 2017, 9060138-11 (2017).

45. Zakrzewski, M. et al. Calypso: a user-friendly web-server for mining and visualizing microbiome-environment interactions. Bioinformatics 33, 782-783 (2017).

46. Caporaso, J. G. et al. QIIME allows analysis of high-throughput community sequencing data. Nat. Methods 7, 335-336 (2010).

47. Hughes, J. B., Hellmann, J. J., Ricketts, T. H. \& Bohannan, B. J. Counting the uncountable: statistical approaches to estimating microbial diversity. Appl. Environ. Microbiol. 67, 4399-4406 (2001).

48. Lozupone, C., Lladser, M. E., Knights, D., Stombaugh, J. \& Knight, R. UniFrac: an effective distance metric for microbial community comparison. The ISME journal 5, 169-172 (2011).

49. Nilsson, R. H. et al. The UNITE database for molecular identification of fungi: handling dark taxa and parallel taxonomic classifications. Nucleic Acids Res. 5, 1-D264 (2018).

50. Faith, J. J. et al. The long-term stability of the human gut microbiota. Science 341, 12374391237439 (2013).

51. Segata, N. et al. Metagenomic biomarker discovery and explanation. Genome Biol. 12, R60 (2011). 